\title{
REDUÇÃO FOTOQUÍMICA DE CROMO HEXAVALENTE POR ÁLCOOIS SOB RADIAÇÃO UV
}

\author{
T. C. MACHADO ${ }^{1}$, J. THEISEN ${ }^{1}$ e M. A. LANSARIN ${ }^{1}$ \\ ${ }^{1}$ Universidade Federal do Rio Grande do Sul, Departamento de Engenharia Química \\ E-mail para contato: tiele@enq.ufrgs.br
}

\begin{abstract}
RESUMO - No presente trabalho se estuda a redução fotoquímica de $\mathrm{Cr}(\mathrm{VI})$ por álcoois, metanol, etanol e propan-2-ol, sob radiação UV. Os experimentos foram realizados em um reator batelada de vidro com controle de temperatura e agitação, sob radiação UV. Primeiramente, avaliou-se a influência do $\mathrm{pH}$ inicial da solução, do volume de álcool e da concentração inicial de cromo hexavalente na redução total de $\mathrm{Cr}(\mathrm{VI})$. Os resultados obtidos permitiram concluir que a redução total de $\mathrm{Cr}(\mathrm{VI})$ é dependente da natureza do álcool, diminuindo na seguinte ordem: propan-2-ol > etanol > metanol. Por fim, realizaram-se experimentos com um efluente industrial, originado em uma indústria de galvanoplastia, tendo sido observados altos valores de redução total de $\mathrm{Cr}(\mathrm{VI})$.
\end{abstract}

\section{INTRODUÇÃO}

O cromo hexavalente é tóxico para a maioria dos organismos, sendo carcinogênico e mutagênico para os animais, podendo causar irritação e corrosão na pele humana. Os metais pesados encontrados no ambiente originam-se, principalmente, da descarga inadequada de efluentes industriais (Ku e Jung, 2001), estando as empresas de curtimento de couro, tintas, corantes, materiais fotográficos, galvanoplastia, mineração, metalúrgicas e de eletrônicos, entre as possíveis fontes de cromo (Schrank et al., 2002; Mohapatra et al., 2005; Chakrabarti et al., 2009). Dentre estas, a indústria de galvanização é caracterizada pelo excessivo consumo de água e energia e pela grande geração de efluentes líquidos contendo metais pesados, tais como, cobre, níquel e cromo. Estes efluentes provêm, principalmente, do descarte das águas de enxágue das peças retiradas dos banhos eletroquímicos.

$\mathrm{O}$ cromo ocorre na natureza em dois estados de oxidação, $\mathrm{Cr}^{+3}$ e $\mathrm{Cr}^{+6}$ ou $\mathrm{Cr}$ (III) e $\mathrm{Cr}$ (VI). O $\mathrm{Cr}(\mathrm{VI})$ é altamente solúvel, o que possibilita a contaminação de lençóis freáticos e outras fontes de água potável, sendo mais estável que o trivalente. A forma hexavalente do cromo é 100 vezes mais tóxica que a trivalente (Mohapatra et al., 2005; Das et al., 2006; Xu et al., 2006; Yang e Chan, 2009; Chakrabarti et al., 2009). Por outro lado, o cromo trivalente é facilmente precipitado e não apresenta toxicidade, sendo considerado um elemento essencial para o metabolismo humano, em níveis controlados (Kim et al., 2002; Mohapatra et al., 2005).

O tratamento convencional para águas residuais contendo $\mathrm{Cr}(\mathrm{VI})$ é a redução com sulfito de sódio ou metabissulfito. Este método, no entanto, apresenta alguns problemas: exige um excesso de 


\section{9 a 22 de outubro de 2014 \\ Florianópolis/SC}

produtos químicos para garantir a conversão completa do $\mathrm{Cr}(\mathrm{VI})$ e ocorre a formação de lama ou a liberação de dióxido de enxofre. Desta maneira, estudos vêm sendo direcionados ao desenvolvimento de tecnologias mais limpas para remediação de efluentes contendo cromo, entre eles, a fotoquímica com álcoois.

A absorção da luz ultravioleta ou visível pela matéria pode promover elétrons para níveis de energia mais altos, gerando espécies eletronicamente excitadas. Esta excitação eletrônica pode ocasionar mudanças químicas, como o desaparecimento de corantes, a fotossíntese em plantas, a degradação/redução de moléculas (Wardle, 2009). A redução fotoquímica de $\mathrm{Cr}$ (VI), na presença de compostos orgânicos e sob radiação UV ou visível, tem sido estudada. Na literatura são encontrados alguns trabalhos sobre a redução fotoquímica de $\mathrm{Cr}$ (VI) por álcoois (Mytych et al., 2003), fenol e seus derivados halogenados (Mytych e Stasicka, 2004) e glicerol (Yurkow et al., 2002). Nestes trabalhos, não há um estudo sistemático e comparativo, que analise o efeito de cada parâmetro da reação, bem como a interação entre eles. Além disso, não foi encontrado nenhum estudo sobre a aplicação de processos fotoquímicos para remediação de $\mathrm{Cr}(\mathrm{VI})$ presente em um efluente de uma indústria Galvânica.

Assim, no presente trabalho foi investigada a redução fotoquímica de cromo hexavalente por álcoois, sob radiação UV. Para avaliar a redução de Cr(VI) por fotoquímica com metanol, etanol e propan-2-ol, foram utilizados planejamentos experimentais que investigaram a influência do $\mathrm{pH}$ inicial da solução, do volume de álcool e da concentração inicial de cromo hexavalente na redução total de Cr(VI). Além disso, realizaram-se experimentos com um efluente industrial, originado em uma indústria Galvânica.

\section{MATERIAIS E MÉTODOS}

Materiais: Dicromato de potássio (Fmaia) foi usado como recebido e água destilada e deionizada foi utilizada para preparar as soluções. Os álcoois etílico absoluto P.A. (Nuclear), metílico P.A. (Química Moderna) e isopropílico P.A. (Dinâmica) foram utilizados nas reações de redução fotoquímica de $\mathrm{Cr}(\mathrm{VI})$.

\subsection{Experimentos de Redução Fotoquímica}

Os ensaios foram realizados em um reator batelada encamisado, irradiado com uma lâmpada de vapor de mercúrio (Philips HPL-N 125 Watt) modificada para emitir na região do UV, pela remoção da camada interna de fósforo do bulbo. A agitação do meio reacional foi mantida por um agitador magnético e a temperatura foi monitorada por um termopar do tipo $\mathrm{K}$ acoplado a um mostrador digital. A câmara de irradiação é constituída por uma caixa de madeira revestida internamente com papel alumínio e um sistema seringa-cateter foi utilizado para coleta de amostras líquidas em tempos determinados. A radiação da lâmpada foi ajustada e medida no início de cada ensaio através de um radiômetro (Cole-Parmer Instrument, Radiometer Series 9811). Uma representação esquemática do reator utilizado é apresentada na Figura 1. 


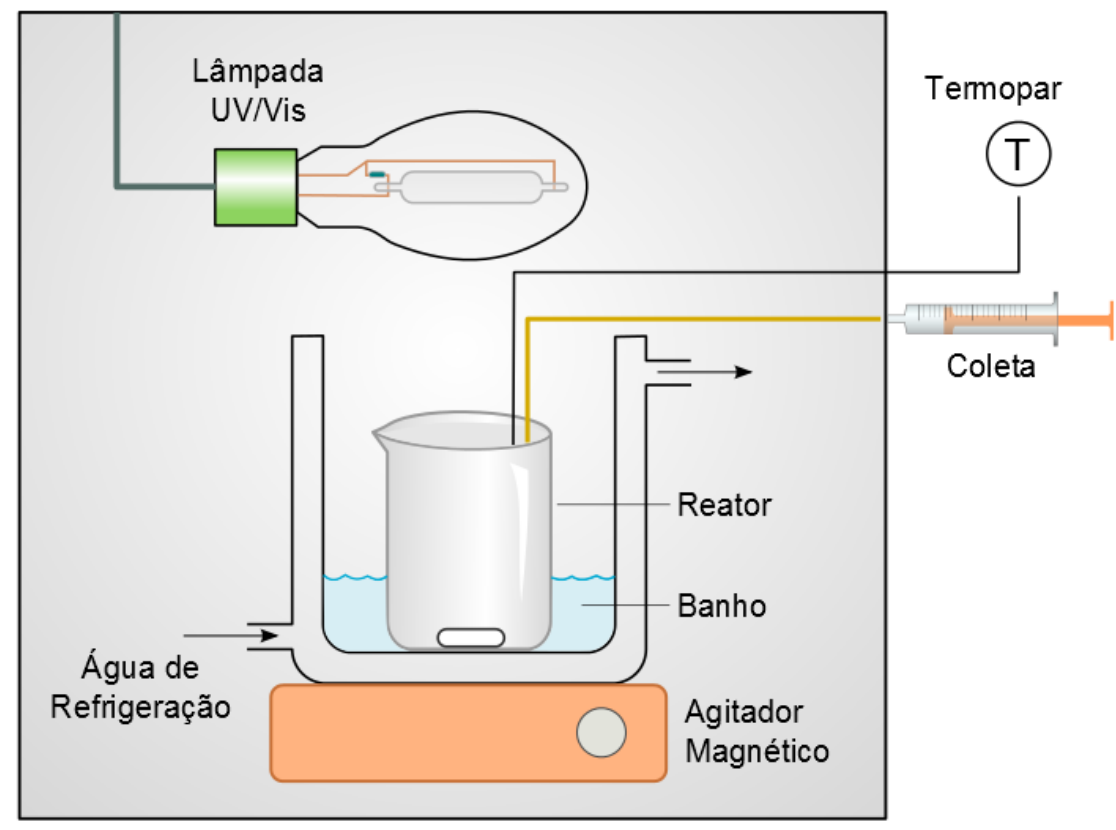

Figura 1 - Figura esquemática do reator usado nos experimentos de fotoquímica.

A solução sintética de cromo hexavalente foi preparada a partir do dicromato de potássio $\left(\mathrm{K}_{2} \mathrm{Cr}_{2} \mathrm{O}_{7}\right)$, sendo mantida sob agitação magnética vigorosa por, aproximadamente, $1 \mathrm{~h}$. Para as reações de redução fotoquímica com álcoois, um volume conhecido de álcool foi adicionado a 150 $\mathrm{mL}$ de solução aquosa de $\mathrm{Cr}(\mathrm{VI})$ e o $\mathrm{pH}$ inicial da solução foi ajustado. Estas reações ocorreram em 1 $\mathrm{h}$, sendo o progresso da reação de redução fotoquímica de $\mathrm{Cr}(\mathrm{VI})$ acompanhado através de coletas de amostras em tempo determinados $(0,15,30,45$ e 60 minuntos). Após as amostras foram analisadas no espectrofotômetro (Varian, Cary 100) através de medidas de absorbância, no comprimento de onda de 348 nm (Mohapatra et al., 2005; Das et al., 2006; Nasrallah et al., 2011). As absorbâncias foram relacionadas com a concentração de $\mathrm{Cr}(\mathrm{VI})$ por uma curva de calibração, de acordo com a Lei de Lambert-Beer.

Nos ensaios realizados com efluente industrial diluído foi utilizada uma solução de cromo hexavalente, originada em uma indústria Galvânica, a qual foi diluída em água destilada e deionizada, a fim de obter a mesma concentração de cromo da melhor condição dos planejamentos de experimentos. Também foram realizados experimentos com efluente industrial na sua concentração original $\left(1100 \mathrm{mg} \mathrm{L}^{-1}\right)$. Estes testes seguiram a mesma metodologia utilizada nas reações com solução sintética de $\mathrm{Cr}(\mathrm{VI})$.

\subsection{Metodologia da Superfície de Resposta}

O modelo estatístico de um planejamento composto central circunscrito (CCC) foi utilizado com o objetivo de otimizar o volume de álcool, o pH inicial da solução e a concentração inicial de $\mathrm{Cr}(\mathrm{VI})$, para as reações de redução fotoquímica de $\mathrm{Cr}(\mathrm{VI})$ sob radiação UV com metanol, etanol e propan-2-ol. Este modelo consiste em um planejamento fatorial completo com $2^{\mathrm{n}}(\mathrm{n}=3)$ 


\section{9 a 22 de outubro de 2014 \\ Florianópolis/SC}

experimentos, 6 pontos estrelas e 3 pontos centrais, dando um total de 17 experimentos. A quantidade de álcool foi variada entre $0,3 \%$ e $6,2 \%, \mathrm{o}$ pH inicial da solução entre 1,5 e 4,0 e a concentração inicial de $\mathrm{Cr}(\mathrm{VI})$ entre $13 \mathrm{mg} \mathrm{L}^{-1}$ e $47 \mathrm{mg} \mathrm{L}^{-1}$.

O fator de resposta, para todos os planejamentos de experimentos, foi definido como sendo a redução total de $\mathrm{Cr}(\mathrm{VI})$, após $1 \mathrm{~h}$ de reação. Os dados foram analisados usando o software Statistica 10, onde foi obtido o polinômio associado ao sistema de reações de redução total de $\mathrm{Cr}(\mathrm{VI})$ e as superfícies de resposta, para cada planejamento experimental. $\mathrm{O}$ modelo foi validado estatisticamente com o mesmo software usando ANOVA (nível de confiança de 95\%).

\section{RESULTADOS E DISCUSSÃO}

\subsection{Reações de Redução Fotoquímica de Cr(VI) com Álcoois}

Com o objetivo de avaliar a influência da natureza do álcool sobre a redução fotoquímica de cromo hexavalente, bem como comparar as reações de redução fotoquímica de $\mathrm{Cr}$ (VI) com diferentes álcoois, foram realizados experimentos de redução fotoquímica de $\mathrm{Cr}(\mathrm{VI})$ com metanol, etanol e propan-2-ol, sob radiação UV. Nestas reações as variáveis estudadas foram o pH inicial da solução, a concentração inicial de $\mathrm{Cr}$ (VI) e o volume de álcool. Na Tabela 1 são apresentados os experimentos de redução fotoquímica de $\mathrm{Cr}$ (VI) com metanol, etanol e propano-2-ol, sob radiação UV. Os números entre parênteses representam as variáveis codificadas e o fator de resposta (Y) foi definido como a percentagem de redução total de Cr(VI) após 1 h de reação.

Os polinômios (Equação (1)-(3)) obtidos a partir da análise multivariada apresentaram um coeficiente de correlação $\left(\mathrm{R}^{2}\right)$ de $0,9913,0,9431$ e 0,9413 para as reações com metanol, etanol e propan-2-ol, respectivamente, indicando um bom ajuste dos dados experimentais aos modelos estatísticos. $\mathrm{O}$ pH inicial da solução e o volume de álcool são as principais variáveis que afetam as reações com etanol e propan-2-ol, com interações quadráticas e lineares, enquanto que, para as reações com metanol, o volume de álcool é a principal variável que afeta estas reações. Nas equações polinomiais, os valores entre parênteses representam o desvio padrão para cada coeficiente e $x, y$ e $z$ representam os valores codificados de $\mathrm{pH}$ inicial da solução, concentração inicial de $\mathrm{Cr}(\mathrm{VI})$ e volume de álcool, respectivamente: $( \pm 0,8)$

$$
Y_{\text {Metanol }}(\%)=40,1( \pm 0,7)-13,9 x( \pm 0,6)-4,3 y( \pm 0,6)+17,2 z( \pm 0,6)-2,9 z^{2}( \pm 0,6)-6,2 x . z
$$

$$
\begin{aligned}
& Y_{\text {Etanol }}(\%)=72,3( \pm 3,8)-25,3 x( \pm 2,6)-11,5 x^{2}( \pm 2,6)-5,8 y( \pm 2,6)+17,1 z( \pm 2,6)-9,0 z^{2}( \pm 2,6) \\
& -11,5 x . z( \pm 3,4) \\
& Y_{\text {Propan-2-ol }}(\%)=72,7( \pm 4,5)-23,7 x( \pm 2,8)-9,4 x^{2}( \pm 2,9)-9,6 y( \pm 2,8)+20,5 z( \pm 2,8)-9,1 z^{2} \\
& ( \pm 2,9)-9,3 x . z( \pm 3,6)
\end{aligned}
$$




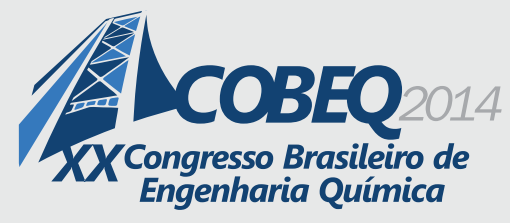

19 a 22 de outubro de 2014

Florianópolis/SC

Tabela 1 - Resultados dos planejamentos experimentais para reação de redução fotoquímica de $\mathrm{Cr}(\mathrm{VI})$ com álcoois sob radiação UV

\begin{tabular}{|c|c|c|c|c|c|c|}
\hline Ensaios & $\begin{array}{c}\text { pH inicial } \\
\text { da solução }\end{array}$ & $\begin{array}{c}\text { Concentração } \\
\text { inicial de Cr(VI) } \\
\left(\mathbf{m g ~ L}^{-\mathbf{1}}\right)\end{array}$ & $\begin{array}{c}\text { Volume de } \\
\text { álcool } \\
(\%)(\mathbf{\%} / \mathbf{v})\end{array}$ & \multicolumn{3}{|c|}{$\begin{array}{c}\text { Redução Total de Cr(VI) } \\
\text { (\%) } \text { (Y }_{\text {exp. }} \text { ) }\end{array}$} \\
\hline 1 & $2(-1)$ & $20(-1)$ & $1,5(-1)$ & 31,5 & 55,5 & 57,3 \\
\hline 2 & $3,5(1)$ & $20(-1)$ & $1,5(-1)$ & 13,5 & 28,9 & 29,9 \\
\hline 3 & $2(-1)$ & $40(1)$ & $1,5(-1)$ & 21,5 & 44,2 & 40,3 \\
\hline 4 & $3,5(1)$ & $40(1)$ & $1,5(-1)$ & 10,2 & 19,3 & 16,8 \\
\hline 5 & $2(-1)$ & $20(-1)$ & $5(1)$ & 79,4 & 96,1 & 98,6 \\
\hline 6 & $3,5(1)$ & $20(-1)$ & $5(1)$ & 41,0 & 34,8 & 55,1 \\
\hline 7 & $2(-1)$ & $40(1)$ & $5(1)$ & 67,6 & 94,9 & 98,2 \\
\hline 8 & $3,5(1)$ & $40(1)$ & $5(1)$ & 27,3 & 12,7 & 16,7 \\
\hline 9 & $1,5(-1,68)$ & $30(0)$ & $3,25(0)$ & 63,8 & 89,5 & 93,9 \\
\hline 10 & $4(1,68)$ & $30(0)$ & $3,25(0)$ & 15,5 & 0,0 & 5,7 \\
\hline 11 & $2,75(0)$ & $13(-1,68)$ & $3,25(0)$ & 46,3 & 86,0 & 89,9 \\
\hline 12 & $2,75(0)$ & $47(1,68)$ & $3,25(0)$ & 34,1 & 65,0 & 53,2 \\
\hline 13 & $2,75(0)$ & $30(0)$ & $0,31(-1,68)$ & 4,5 & 9,3 & 4,2 \\
\hline 14 & $2,75(0)$ & $30(0)$ & $6,2(1,68)$ & 61,8 & 94,5 & 96,8 \\
\hline 15 & $2,75(0)$ & $30(0)$ & $3,25(0)$ & 43,0 & 71,4 & 73,7 \\
\hline 16 & $2,75(0)$ & $30(0)$ & $3,25(0)$ & 39,1 & 74,0 & 76,2 \\
\hline 17 & $2,75(0)$ & $30(0)$ & $3,25(0)$ & 43,1 & 71,6 & 76,8 \\
\hline
\end{tabular}

Nas Equações (1)-(3), pode-se observar, para todas as reações fotoquímicas (metanol, etanol e propan-2-ol), que os coeficientes referentes aos efeitos lineares do $\mathrm{pH}$ e da concentração de $\mathrm{Cr}(\mathrm{VI})$ apresentaram valores negativos, indicando que quanto menor for o $\mathrm{pH}$ inicial da solução e a concentração inicial de $\mathrm{Cr}(\mathrm{VI})$, maior será a redução total de $\mathrm{Cr}(\mathrm{VI})$, enquanto que o valor do coeficiente referente ao efeito linear do volume de álcool foi positivo, logo quanto maior for o volume de álcool, maior será a redução total de $\mathrm{Cr}(\mathrm{VI})$. Este comportamento pode ser observado na Tabela 1, onde os ensaios 5, para as reações com metanol, etanol e propan-2-ol, com menores valores de $\mathrm{pH}$ inicial da solução e concentração inicial de $\mathrm{Cr}(\mathrm{VI})$ e maior valor de volume de álcool, apresentaram as maiores reduções totais de $\mathrm{Cr}(\mathrm{VI})$ de 79,4\%, 96,1\% e 98,6\%, respectivamente. Maiores informações a respeito das reações de redução fotoquímica de $\mathrm{Cr}(\mathrm{VI})$ com etanol sob radiação UV podem ser encontradas no trabalho de Machado et al. (2014).

Mytych et al. (2003) estudaram a redução fotoquímica de Cr(VI) mediada por álcoois alifáticos e observaram que a taxa da reação é dependente da natureza do álcool diminuindo na seguinte ordem: butan-1-ol > butan-2-ol >> propan-2-ol > etanol >> metanol > 2-metil-propan-2-ol. Comportamento semelhante foi verificado neste trabalho, onde os resultados das reações de redução fotoquímica de $\mathrm{Cr}(\mathrm{VI})$ com metanol, etanol e propan-2-ol, sob radiação UV, nas condições ótimas dos planejamentos experimentais, estão apresentados na Figura 2. Como pode ser observado nesta Figura, o álcool isopropílico apresentou uma redução total de $\operatorname{Cr}(\mathrm{VI})$ de $98,6 \%$, enquanto que o álcool etílico 
apresentou uma redução de $96,1 \%$. No entanto, considerando o erro absoluto dos experimentos, os valores de redução total para o etanol e o propan-2-ol são iguais.

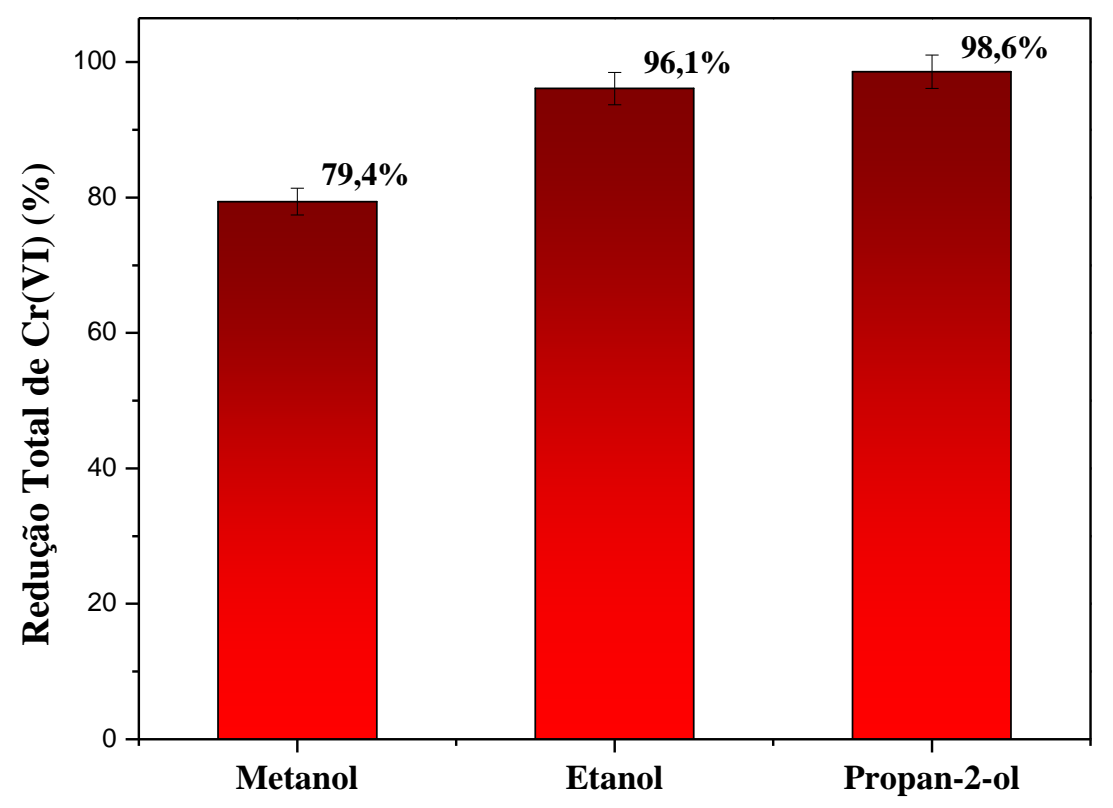

Figura 2 - Comparação das reações de redução fotoquímica de $\mathrm{Cr}(\mathrm{VI})$ com diferentes álcoois, sob radiação $\mathrm{UV}\left(\mathrm{C}_{\mathrm{Cr}(\mathrm{VI})}=20 \mathrm{mg} \mathrm{L}^{-1}, \mathrm{~V}_{\text {álcool }}=5,0 \%(\mathrm{v} / \mathrm{v}), \mathrm{T}=30^{\circ} \mathrm{C}, \mathrm{pH}=2,0\right.$ e $\left.5,8 \mathrm{~mW} \mathrm{~cm}^{-2}\right)$.

\subsection{Ensaios com Efluente Industrial}

Com intuito de verificar a aplicação da fotoquímica com álcoois para remediação do cromo hexavalente presente no efluente de uma indústria Galvânica foram realizados ensaios de redução total de $\mathrm{Cr}(\mathrm{VI})$ com o efluente na sua concentração original (1100 $\left.\mathrm{mg} \mathrm{L}^{-1}\right)$. Para isto, utilizou-se um volume de álcool de $40 \%$ (v/v) e um tempo de reação de 6 horas. Os resultados são apresentados na Tabela 2.

Pode-se observar, na Tabela 2, que o processo de redução fotoquímica de $\mathrm{Cr}(\mathrm{VI})$ com isopropanol obteve uma redução total de $\mathrm{Cr}(\mathrm{VI})$ igual à obtida para fotoquímica com etanol, possibilitando o uso destes processos para o tratamento de efluentes de indústrias Galvânicas. No entanto, dentre os álcoois estudados, o etanol foi considerado o mais suscetível para aplicação industrial, devido ao seu baixo custo, não toxicidade e facilidade de aquisição (Machado et al., 2014). 
Tabela 2 - Ensaios de redução total de Cr(VI) com efluente industrial na sua concentração original

\begin{tabular}{|c|c|c|}
\hline Reações & Condições Experimentais & Redução Total de $\operatorname{Cr}(\mathrm{VI})(\%)$ \\
\hline Fotoquímica Etanol & $\begin{array}{c}\mathrm{C}_{\mathrm{Cr}(\mathrm{VI})}=1100 \mathrm{mg} \mathrm{L}^{-1}, \mathrm{pH}=2,0, \\
\mathrm{~V}_{\text {etanol }}=40 \% \text { e } 5,8 \mathrm{~mW} \mathrm{~cm} \mathrm{~cm}^{-2} \\
6 \text { horas de reação }\end{array}$ & 98,48 \\
\hline Fotoquímica Metanol & 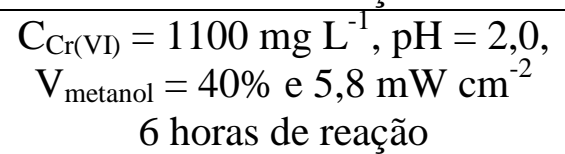 & 68,88 \\
\hline Fotoquímica Propan-2-ol & 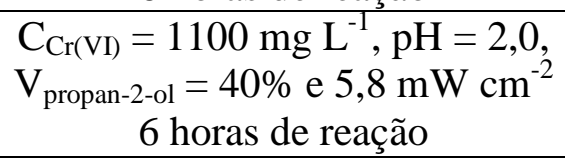 & 98,97 \\
\hline
\end{tabular}

\section{CONCLUSÕES}

Os resultados obtidos permitiram concluir que as reações de redução fotoquímica de $\mathrm{Cr}(\mathrm{VI})$ com álcoois, sob radiação UV, são dependentes do pH inicial da solução, da concentração inicial de $\mathrm{Cr}(\mathrm{VI})$ e do volume de álcool. Para estas reações, a redução total de $\mathrm{Cr}(\mathrm{VI})$ diminui com o aumento do pH inicial da solução e da concentração inicial de $\mathrm{Cr}(\mathrm{VI})$ e aumenta com aumento do volume de álcool. Além disso, a redução total de $\mathrm{Cr}(\mathrm{VI})$ mostrou-se dependente da natureza do álcool, diminuindo na seguinte ordem: propan-2-ol > etanol $\gg$ metanol. Ainda, os resultados obtidos para os experimentos realizados com efluente industrial, originado em uma indústria Galvânica, mostraram que, dentre os álcoois estudados, o etanol é o mais suscetível para aplicação industrial, devido ao seu baixo custo, não toxicidade e facilidade de aquisição.

\section{AGRADECIMENTOS}

Os autores agradecem o auxílio financeiro da CAPES e do CNPq para realização deste trabalho.

\section{REFERENCIAS}

CHAKRABARTI, S.; CHAUDHURI, B.; BHATTACHARJEE, S.; RAY. A. K.; DUTTA, B. K. Photo-reduction of hexavalent chromium in aqueous solution in the presence of zinc oxide as semiconductor catalyst. Chem. Eng. J., v. 153, p. 86-93, 2009.

DAS, D. P.; PARIDA, K.; DE, B. R. Photocatalytic reduction of hexavalent chromium in aqueous solution over titania pillared zirconium phosphate and titanium phosphate under solar radiation. 
J. Mol. Catal. A Chem., v. 245, p. 217-224, 2006.

KIM, S. D.; PARK, K. S.; GU, M. B. Toxicity of hexavalent chromium to Daphnia magna: influence of reduction reaction by ferrous iron. J. Hazard. Mater., v. A93, p. 155-164, 2002.

KU, Y.; JUNG, I-L. Photocatalytic reduction of $\mathrm{Cr}(\mathrm{VI})$ in aqueous solutions by UV irradiation with the presence of titanium dioxide. Water Res., v. 35, p. 135-142, 2001.

MACHADO, T. C.; LANSARIN, M. A.; MATTE, N. Reduction of hexavalent chromium: photocatalysis and photochemistry and its application in wastewater remediation. Water Sci. Technol., in press, 2014.

MOHAPATRA, P.; SAMANTARAY, S. K.; PARIDA, K. Photocatalytic reduction of hexavalent chromium in aqueous solution over sulphate modified titania. J. Photochem. Photobiol. A, v. 170, p. 189-194, 2005.

MYTYCH, P.; KAROCKI, A.; STASICKA, Z. Mechanism of photochemical reduction of chromium(VI) by alcohols and its environmental aspects. J. Photochem. Photobiol. A, v. 160, p. 163-170, 2003.

MYTYCH, P.; STASICKA, Z. Photochemical reduction of chromium(VI) by phenol and its halogen derivatives. Appl. Catal. B, v. 52, p. 167-172, 2004.

NASRALLAH, N.; KEBIR, M.; KOUDRI, Z.; TRARI, M. Photocatalytic reduction of Cr(VI) on the novel hetero-system CuFe2O4/CdS. J. Hazard. Mater., v. 185, p. 1398-1404, 2011.

SCHRANK, S. G.; JOSÉ, H. J.; MOREIRA, R. F. P. M. Simultaneous photocatalytic Cr(VI) reduction and dye oxidation in a TiO2 slurry reactor. J. Photochem. Photobiol. A, v. 147, p. 7176, 2002.

WARDLE, B. Principles and applications of photochemistry. John Wiley and Sons Ltd, Manchester Metropolitan University, Manchester, UK, 2009.

XU, X.; LI, H.; GU, J. Simultaneous decontamination of hexavalent chromium and methyl tert-butyl ether by UV/TiO2 process. Chemosphere, v. 63, p.254-260, 2006.

YANG, G. C. C.; CHAN, S-W. Photocatalytic reduction of chromium(VI) in aqueous solution using dye-sensitized nanoscale $\mathrm{ZnO}$ under visible light irradiation. J. Nanopart. Res., v. 11, p. 221230, 2009.

YURKOW, E. J.; HONG, J.; MIN, S.; WANG, S. Photochemical reduction of hexavalent chromium in glycerol-containing solutions. Environ. Pollut., v. 117, p. 1-3, 2002. 